\title{
An efficient approach of the pseudospectral method for modelling of geometrically symmetric seismic wavefield
}

\author{
Hiroshi Takenaka ${ }^{1}$, Yanbin Wang ${ }^{1}$, and Takashi Furumura ${ }^{2}$ \\ ${ }^{1}$ Department of Earth and Planetary Sciences, Faculty of Science, Kyushu University, Hakozaki 6-10-1, Fukuoka 812-8581, Japan \\ ${ }^{2}$ Faculty of Education, Hokkaido University of Education, Midorigaoka 2-34-1, Iwamizawa 068-0835, Japan
}

(Received July 7, 1998; Revised October 30, 1998; Accepted December 9, 1998)

\begin{abstract}
The pseudospectral method is a high-accuracy numerical modelling technique that requires less computer memory and computation time than the traditional techniques such as the finite-difference method. These advantages of the pseudospectral method have enabled us to practically apply this method to modelling realistic problems that have complex structure and source models. However, a major drawback of such numerical schemes for discrete grid models is that even for rather a simple structural model they require as much computational requirements (e.g. computation time and memory) as for an entirely complex structural model with the same size of the simple one. We actually need to employ idealised simple models, such as a model with geometrical symmetry, to investigate basic phenomena of seismic waves, to develop new techniques, or to choose optimal values of some computational parameters for more complex modelling. In this paper we propose an efficient approach of an economical pseudospectral method for calculation of wavefields in models symmetric with respect to a vertical plane or two orthogonal vertical planes. Using this approach, the wavefields only need to be computed in a half or quarter domain of the models, so that the computer memory and computation time can be reduced ideally by half or quarter, respectively, as compared with the calculation of the entire models.
\end{abstract}

\section{Introduction}

The pseudospectral method (the Fourier method) (e.g., Kosloff et al., 1984; Reshef et al., 1988; Furumura et al., 1998 ) is an attractive alternative to numerical modelling schemes such as the finite difference or finite element method. In the pseudospectral method, the field quantities are expanded in the space in terms of Fourier interpolation polynomials. Numerical differentiation in the equations with respect to the spatial coordinates is then implemented via the discrete Fourier transform (the fast Fourier transform). Since no approximation of the spatial derivatives is involved in the differentiation scheme, fewer grid points are required to achieve computational accuracy. The accurate spatial differentiation can reduce computer memory and computation time by several orders of magnitude as compared with other numerical methods such as the finite difference or finite element method (e.g., Fornberg, 1987; Daudt et al., 1989).

While efficient numerical methods such as the pseudospectral method can simulate the wave propagation in realistic complex structures (e.g., Furumura and Koketsu, 1998), we also often use rather idealised simple models, such as a model with geometrical symmetry, to investigate basic phenomena of seismic waves, to develop new techniques (e.g., a new non-reflecting boundary condition), to check a new method or new code, or to find optimal values of some computational parameters (e.g., grid size, parameters for absorbing boundary condition) in preparation for more complex modelling. When geometrical symmetry is present in the model, explicit

Copy right (C) The Society of Geomagnetism and Earth, Planetary and Space Sciences (SGEPSS); The Seismological Society of Japan; The Volcanological Society of Japan; The Geodetic Society of Japan; The Japanese Society for Planetary Sciences. use of this symmetry can reduce the computational size to save several factors of magnitude in both computer memory and computation time, because the wavefield need to be calculated only in a subdomain of the model, determined by the type of symmetry, instead of the entire model (e.g., Boore, 1972).

In this paper we propose an efficient approach of the pseudospectral method for calculation of elastic wavefield in a model where the configuration of medium and source is symmetric with respect to a vertical plane or two orthogonal vertical planes. Throughout this paper we employ a right handed Cartesian coordinate system $[x, y, z]$ with $z$ vertically downwards. We will then consider a model symmetric with respect to the $y-z$ plane or the $y-z$ and $x-z$ planes. In this paper we consider only an isotropic elastic medium, while our approach is also applicable to anisotropic viscoelastic cases.

\section{Pseudospectral Modelling for Elastic Waves}

Here we briefly explain the pseudospectral calculation of 3-D elastic waves. The equation of motion is represented as

$$
\begin{aligned}
& \rho \ddot{u}=\frac{\partial \sigma_{x x}}{\partial x}+\frac{\partial \sigma_{x y}}{\partial y}+\frac{\partial \sigma_{z x}}{\partial z}+f_{x}, \\
& \rho \ddot{v}=\frac{\partial \sigma_{x y}}{\partial x}+\frac{\partial \sigma_{y y}}{\partial y}+\frac{\partial \sigma_{y z}}{\partial z}+f_{y}, \\
& \rho \ddot{w}=\frac{\partial \sigma_{z x}}{\partial x}+\frac{\partial \sigma_{y z}}{\partial y}+\frac{\partial \sigma_{z z}}{\partial z}+f_{z},
\end{aligned}
$$

where $[u, v, w]=\boldsymbol{u}$ are the displacements, $[\ddot{u}, \ddot{v}, \ddot{w}]=\ddot{\boldsymbol{u}}$ are the second partial time derivatives of the displacements (i.e., particle accelerations), $\sigma_{p q},(p, q=x, y, z)$ are stress 
components, $f_{p}$ are body forces, and $\rho$ is the density. In an isotropic elastic medium, the stress components are given by

$$
\begin{array}{r}
\sigma_{p q}=\lambda\left(e_{x x}+e_{y y}+e_{z z}\right) \delta_{p q}+2 \mu e_{p q}, \\
(p, q=x, y, z)
\end{array}
$$

where $\lambda$ and $\mu$ are the Lamé constants, $e_{p q}$ are the strain components, and $\delta_{p q}$ denotes Kronecker's delta. The strain components are defined as

$$
\begin{gathered}
e_{x x}=\frac{\partial u}{\partial x}, \quad e_{y y}=\frac{\partial v}{\partial y}, \quad e_{z z}=\frac{\partial w}{\partial z} \\
e_{y z}=\frac{1}{2}\left(\frac{\partial v}{\partial z}+\frac{\partial w}{\partial y}\right), \quad e_{z x}=\frac{1}{2}\left(\frac{\partial w}{\partial x}+\frac{\partial u}{\partial z}\right), \\
e_{x y}=\frac{1}{2}\left(\frac{\partial u}{\partial y}+\frac{\partial v}{\partial x}\right) .
\end{gathered}
$$

In the pseudospectral method the spatial derivatives in Eqs. (1) and (3) are calculated analytically in the wavenumber domain by use of the fast Fourier transform (FFT). The FFT for the transform of complex-valued data (i.e., complexFFT) is often used in the pseudospectral modelling, but such a transformation and the differentiation for originally realvalued data can also be calculated efficiently by use of the real-FFT (e.g., see Furumura et al., 1998) or the Hartley transform (e.g., see Saatcilar and Ergintav, 1991).

For the time evaluation, an explicit scheme is used (the wavefield at the next time step is calculated using the current and previous wavefields). For example, the following second-order finite difference time integration scheme is often used:

$$
\dot{\boldsymbol{u}}^{n+1 / 2}=\dot{\boldsymbol{u}}^{n-1 / 2}+\ddot{\boldsymbol{u}}^{n} \Delta t
$$

and

$$
\boldsymbol{u}^{n+1}=\boldsymbol{u}^{n-1}+\dot{\boldsymbol{u}}^{n+1 / 2} \Delta t,
$$

where $\Delta t$ is the time increment, and $\dot{\boldsymbol{u}}^{n+1 / 2}$ are the particle velocities at time $t=(n+1 / 2) \Delta t$.

\section{Symmetry of Elastic Wavefield}

Now we consider elastic wavefield in a model where the configuration of medium and source is symmetric with respect to a vertical plane. For simplicity, we here confine our attention to 2-D $P-S V$ modelling, where the $y$-coordinate will be suppressed. The cases of 3-D modelling will be outlined in Section 6.

Consider a structure model whose medium parameters are symmetric with respect to the plane $x=0$ ( $y$-z plane $)$, i.e.

$$
\begin{gathered}
\lambda(-x, \cdot)=\lambda(x, \cdot), \quad \mu(-x, \cdot)=\mu(x, \cdot), \\
\rho(-x, \cdot)=\rho(x, \cdot),
\end{gathered}
$$

$\lambda, \mu$ and $\rho$ are even functions of $x$, and the vertical coordinate $z$ has been replaced by the dot '. ' to focus on the $x$-coordinate.

Then, the displacement field $[u(x, z), w(x, z)]$ excited by vertical incidence of a plane wave or source with force system either symmetric or anti-symmetric in the $x$ coordinate, which is distributed symmetrically with respect to the plane $x=0$, has the following symmetric or anti-symmetric property: for vertical incidence of a plane $P$ wave, or source with force system symmetric in the $x$ coordinate, such as that for the single force $f_{z}$ and moment tensor components $M_{x x}$ and $M_{z z}$ with all other components being zero,

$$
\begin{gathered}
u(-x, \cdot)=-u(x, \cdot) \\
w(-x, \cdot)=w(x, \cdot) \\
\frac{\partial u}{\partial x}(-x, \cdot)=\frac{\partial u}{\partial x}(x, \cdot), \quad \frac{\partial u}{\partial z}(-x, \cdot)=-\frac{\partial u}{\partial z}(x, \cdot), \\
\frac{\partial w}{\partial x}(-x, \cdot)=-\frac{\partial w}{\partial x}(x, \cdot), \quad \frac{\partial w}{\partial z}(-x, \cdot)=\frac{\partial w}{\partial z}(x, \cdot) ;
\end{gathered}
$$

while for vertical incidence of a plane $S V$ wave, or source with force system anti-symmetric in $x$, such as that for the single force $f_{x}$ and moment tensor components $M_{x z}$ and $M_{z x}$ with all other components being zero,

$$
\begin{gathered}
u(-x, \cdot)=u(x, \cdot), \\
w(-x, \cdot)=-w(x, \cdot), \\
\frac{\partial u}{\partial x}(-x, \cdot)=-\frac{\partial u}{\partial x}(x, \cdot), \quad \frac{\partial u}{\partial z}(-x, \cdot)=\frac{\partial u}{\partial z}(x, \cdot), \\
\frac{\partial w}{\partial x}(-x, \cdot)=\frac{\partial w}{\partial x}(x, \cdot), \quad \frac{\partial w}{\partial z}(-x, \cdot)=-\frac{\partial w}{\partial z}(x, \cdot),
\end{gathered}
$$

where the time dependence of the field quantities has been suppressed in these equations. Equations (7) to (14) show each component of the displacement and its spatial derivatives are even or odd functions of $x$. Note that differentiation with respect to $z$ mapps odd/even functions of $x$ into odd/even functions of $x$, while differentiation with respect to $x$ mapps odd/even functions of $x$ into even/odd functions.

The stress components $\sigma_{i j}(x, z)(i, j=x$ or $z)$ then have symmetric or anti-symmetric property with $x$, as follows:

$$
\begin{gathered}
\sigma_{x x}(-x, \cdot)=\sigma_{x x}(x, \cdot), \\
\sigma_{z z}(-x, \cdot)=\sigma_{z z}(x, \cdot), \\
\sigma_{z x}(-x, \cdot)=-\sigma_{z x}(x, \cdot),
\end{gathered}
$$

for vertical incidence of a plane $P$ wave, or source with force system symmetric in $x$;

$$
\begin{gathered}
\sigma_{x x}(-x, \cdot)=-\sigma_{x x}(x, \cdot), \\
\sigma_{z z}(-x, \cdot)=-\sigma_{z z}(x, \cdot), \\
\sigma_{z x}(-x, \cdot)=\sigma_{z x}(x, \cdot),
\end{gathered}
$$

for vertical incidence of a plane $S V$ wave, or source with force system anti-symmetric in $x$. Equations (15) to (20) can be derived from Eqs. (6), (9), (10), (13) and (14) and the relation between the stress and displacement (i.e., Hooke's law).

Equations (7) to (20) suggest that the wavefield need to be calculated only in the left half domain $x<0$ or the right half domain $x>0$ of the model (half-domain modelling) instead of the full domain of the model (full-domain modelling), which reduces the computational size by half in the numerical calculation. In the next section we will describe schemes for calculating spatial derivatives only using the right half domain of the model. 


\section{Spatial Differentiation Using the FFT}

In the pseudospectral method the spatial differentiation is efficiently calculated at each time step by means of the FFT as follows. For instance, consider the $x$-differentiation of the $x$-component of the displacement at the spatially discretised locations, $u((n+1 / 2) \Delta x,(m+1 / 2) \Delta z)(n=-N,-N+$ $1, \ldots, 0, \ldots, N-1 ; m=0,1, \ldots, M-1)$, where the model consists of $2 N \times M$ grid points, and the $x$-derivative to be calculated is $\partial u / \partial x((n+1 / 2) \Delta x,(m+1 / 2) \Delta z)(n=$ $-N,-N+1, \ldots, 0, \ldots, N-1 ; m=0,1, \ldots, M-1)$.

First, $u((n+1 / 2) \Delta x, \Delta z / 2)(n=-N,-N+1, \ldots, 0$, $\ldots, N-1)$, is copied into a 1-D work array (work vector) $f(n)(n=-N,-N+1, \ldots, 0, \ldots, N-1)$. Second, $f(n)$ $(n=-N,-N+1, \ldots, 0, \ldots, N-1)$ is transformed to the wavenumber domain by use of a 1-D FFT. The result is then multiplied by the discrete spatial wavenumbers and the imaginary unit to obtain the derivative in the wavenumber domain and transformed back to the physical domain using an inverse 1-D FFT. The final result is copied into $\partial u / \partial x((n+$ $1 / 2) \Delta x, \Delta z / 2)(n=-N,-N+1, \ldots, 0, \ldots, N-1)$. Next, $u((n+1 / 2) \Delta x, 3 \Delta z / 2)$ is processed in the same way as $u((n+1 / 2) \Delta x, \Delta z / 2)$ to get $\partial u / \partial x((n+1 / 2) \Delta x, 3 \Delta z / 2)$, and the same process is carried out sequentially with increasing $z$ in the interval of $\Delta z$ up to $z=(M-1 / 2) \Delta z$.

This is a usual scheme for the $x$-differentiation of 2-D field quantities by a 1-D FFT (see for detail, e.g. Furumura et al., 1998). Hereafter we call this scheme the normal Fourier differentiation scheme. The $z$-differentiation can be computed in the similar way using this normal Fourier differentiation scheme.

When $u(x, z)$ is an odd function of $x$ (i.e. Eq. (7)) or even function of $x$ (i.e. Eq. (11)), the values only for the right half domain $x>0$ of the model are necessary at each time step of the pseudospectral calculation, because the other half domain $(x<0)$ can naturally be given by the symmetric and anti-symmetric relations in the wavefield with respect to $x=0$. The $x$-differentiation can then be performed by the following scheme including a usual Fourier differentiation process mentioned above, so that we need little modification on our available pseudospectral codes.

First, $u((n+1 / 2) \Delta x, \cdot)(n=0, \ldots, N-1)$ is copied into the latter half of a work vector of length $2 N$. The first half of this vector is assigned the values for $x<0$ using Eq. (7) or Eq. (11). That is, when $u(x, z)$ is odd in $x$, the first half of the vector is given the values along Eq. (7) (hereafter called anti-symmetric extension) as:

$$
f(n)=\left\{\begin{array}{c}
-u(-(n+1 / 2) \Delta x, \cdot) \\
(n=-N,-N+1, \ldots,-1), \\
u((n+1 / 2) \Delta x, \cdot) \\
\quad(n=0,1, \ldots, N-1) ;
\end{array}\right.
$$

when $u(x, z)$ is even in $x$, the first half of the vector is given the values along Eq. (11) (symmetric extension) as:

$$
f(n)=\left\{\begin{array}{c}
u(-(n+1 / 2) \Delta x, \cdot) \\
(n=-N, N+1, \ldots,-1), \\
u((n+1 / 2) \Delta x, \cdot) \\
(n=0,1, \ldots, N-1) .
\end{array}\right.
$$

And a usual Fourier differentiation is then done on this vector. The latter half of the resultant vector is copied into $\partial u / \partial x((n+1 / 2) \Delta x, \cdot)(n=0,1, \ldots, N-1)$ and the remaining half of the data $(n=-N, N+1, \ldots,-1)$ is abandoned.

This Fourier differentiation scheme with symmetric or anti-symmetric extension is applied to calculate the $x$ derivatives appearing in Eqs. (1) and (3), while $z$-derivatives are computed by use of the normal Fourier differentiation scheme because of no symmetry in $z$.

\section{Numerical Example}

In order to demonstrate the feasibility of our approach mentioned above, we calculate the wavefield for a simple 2-D semi-cylindrical sedimentary basin in case of $S V$ plane-wave vertical incidence both by full- and half-domain modellings, and then compare their results. The model is depicted in Fig. 1 where a semi-cylindrical basin with radius $a=2.5$ $\mathrm{km}$ is embedded in a homogeneous half-space. $P$-, $S$-wave velocity and density of the basin are $4.0 \mathrm{~km} / \mathrm{s}, 2.0 \mathrm{~km} / \mathrm{s}$ and $2.0 \mathrm{~g} / \mathrm{cm}^{3}$, and those of the half-space are $6.9 \mathrm{~km} / \mathrm{s}, 4.0 \mathrm{~km} / \mathrm{s}$ and $2.6 \mathrm{~g} / \mathrm{cm}^{3}$, respectively. Input signal is a Ricker wavelet with the central frequency of $1.2 \mathrm{~Hz}$, which implies the wavelength of input wave is equal to the diameter of the basin.

The computational domain for full-domain modelling is $51.2 \mathrm{~km}$ long and $12.8 \mathrm{~km}$ deep as shown in Fig. 2(a). Since the geometry of the model and the input plane wave are symmetric about $z$ axis, the wavefield of the right half domain $x>0$ (Fig. 2(b)) can be calculated by half-domain modelling, which can then be extended by the symmetric and anti-symmetric extensions to get the entire wavefield. In this example the relation for the extension is given by Eqs. (11) and (12). In half-domain modelling of this example the $x$-derivatives are calculated by the above-mentioned Fourier differentiation scheme with the symmetric or anti-symmetric extension as given in Eqs. (11), (12) and (18) to (20), while the $z$-derivatives are computed by the normal Fourier differentiation scheme.

For both full- and half-domain modellings, we use the grid intervals of $0.1 \mathrm{~km}$ in horizontal and vertical dimensions. Figures 2(a) and (b) also show the grid layouts around the basin for full- and half-domain modellings, respectively. For the time evaluation, we employ the second-order finite difference time integration scheme. The time increment of $0.0025 \mathrm{~s}$, and the total number of time steps is 4000 , so that the calculated time window is $10 \mathrm{~s}$. The absorbing boundary (Cerjan et al., 1985) of 20 grid size is applied to the bottom of

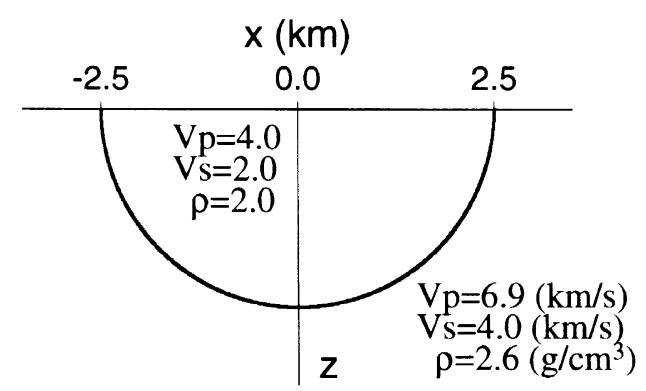

Fig. 1. Model configuration. A semi-cylindrical basin is embedded in a homogeneous half-space. 
(a) full-domain modelling
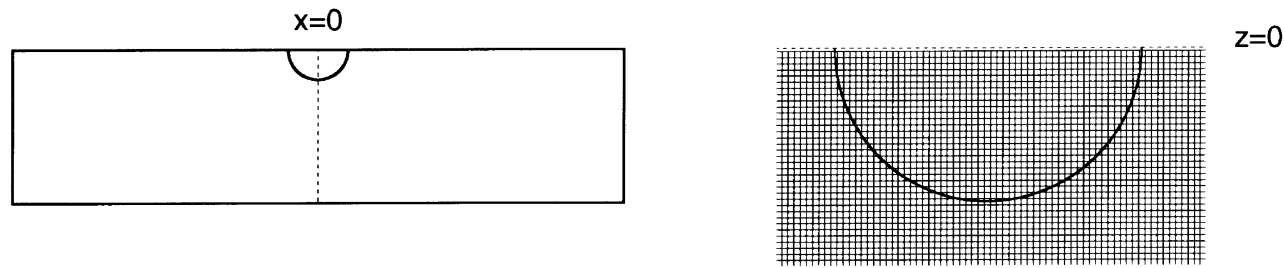

(b) half-domain modelling
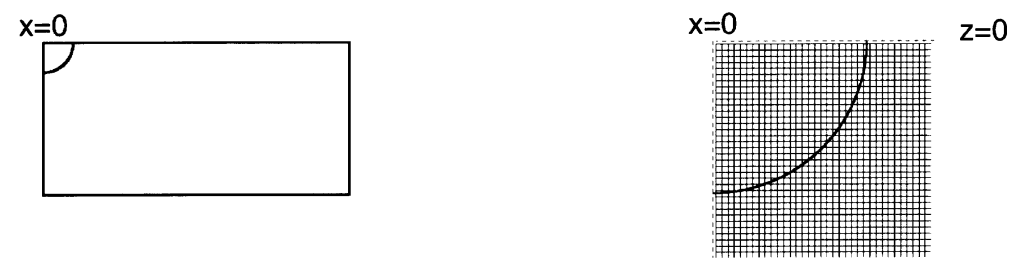

Fig. 2. (a) Computational domain for full-domain modelling, which is symmetric with respect to $x=0$ and $51.2 \mathrm{~km}$ long and $12.8 \mathrm{~km}$ deep (left), and the grid layout around the basin (right). (b) Computational domain for half-domain modelling whose size is $25.6 \mathrm{~km}$ long and $12.8 \mathrm{~km}$ deep (left), and the grid layout around the basin (right).

the computational domains to suppress wraparound due to the spatial periodicity implicitly involved in the FFT. The freesurface condition is incorporated in the calculation by adding a number of zeros to the stress components above the free surface prior to their vertical $(z)$ differentiations (Furumura and Takenaka, 1992), so that the free surface $(z=0)$ is at the level of a half grid size above the top of the discretised domains of the model (Figs. 2(a), (b)).

Figure 3 shows the synthetic seismograms along the level that is a half grid size below the free surface. Vertical axis $x / a$ is the relative distance of observation stations from the center of the basin: the stations at $x / a$ between -1 and 1 are at the top of the basin and the other stations are at the top of the half-space. Figure 3(a) is the horizontal $(u)$ and vertical $(w)$ components calculated directly by full-domain modelling, while the seismograms in Fig. 3(b) have been calculated for the stations with $x / a>0$ by half-domain modelling and then extended by Eqs. (11) and (12) to get the other seismograms $(x / a<0)$. The direct $S V$ wave, refracted $S V$ and $P$ waves from the basin boundary, reflected $S V$ and $P$ waves from the basin surface, and Rayleigh waves generated at the basin edge are observed to appear at exactly the same time with the same amplitudes in Figs. 3(a) and 3(b).

Figure 4 shows the snapshots of wavefield inside and around the basin at $t=0.5,1.0$ and $3.0 \mathrm{~s}$. The snapshots are represented by the $P$-wave and $S V$-wave contributions that are shown in white and black, respectively. Contributions from $P$ and $S V$ waves are calculated from the divergence and curl of the wavefield:

$$
\begin{gathered}
P:\left|\frac{\partial u}{\partial x}+\frac{\partial w}{\partial z}\right|, \\
S V:\left|\frac{\partial w}{\partial x}-\frac{\partial u}{\partial z}\right|
\end{gathered}
$$

The extension of these $P$ and $S V$ wavefields obtained by half-domain modelling to the entire domain has been made by Eqs. (13) and (14). Figure 4(a) is the result directly calculated by full-domain modelling, where the refracted $S V$ wave from the basin boundary, reflected $S V$ and $P$ waves from the surface and the basin-edge generated Rayleigh wave (denoted by " $R$ ") can be observed inside the basin. Figure 4(b) is the result of half-domain modelling, and Fig. 4(c) is the entire wavefield derived by its extension based on Eqs. (13) and (14). Comparing Figs. 4(a) and (c), the wavefields calculated by full-domain modelling and by extension of the solution of half-domain modelling are exactly the same.

In this example, the number of grid points for full- and halfdomain modellings is $512 \times 128$ and $256 \times 128$, respectively, and the total number of time steps are 4000 . The computer memory required by full- and half-domain modellings is then 5.72 Mbytes and 2.89 Mbytes, respectively, using singleprecision arithmetic. On a SUN Ultra-1 workstation (Model $140,143 \mathrm{MHz}$ ), the CPU time for half-domain modelling was $269.9 \mathrm{~min}$, while for full-domain modelling was $415.9 \mathrm{~min}$. Half-domain modelling could save the computer memory and CPU time by $50 \%$ and $35 \%$, respectively, as compared with full-domain modelling. Such efficiency would be remarkable for larger scale and more complex model which requires much larger computer memory and longer CPU time.

\section{3-D Cases}

We now sketch the approach for 3-D cases with symmetry about a vertical plane or two orthogonal vertical planes $(y-z$ and $z-x$ planes) (2-D cases can also be derived from the $3-\mathrm{D}$ cases by setting the derivatives with respect to $y$ to be all zero).

For any model symmetric with respect to a vertical plane, the wavefield only need to be computed in a half domain of 


\section{(a) full-domain modelling}

$\mathrm{u}$

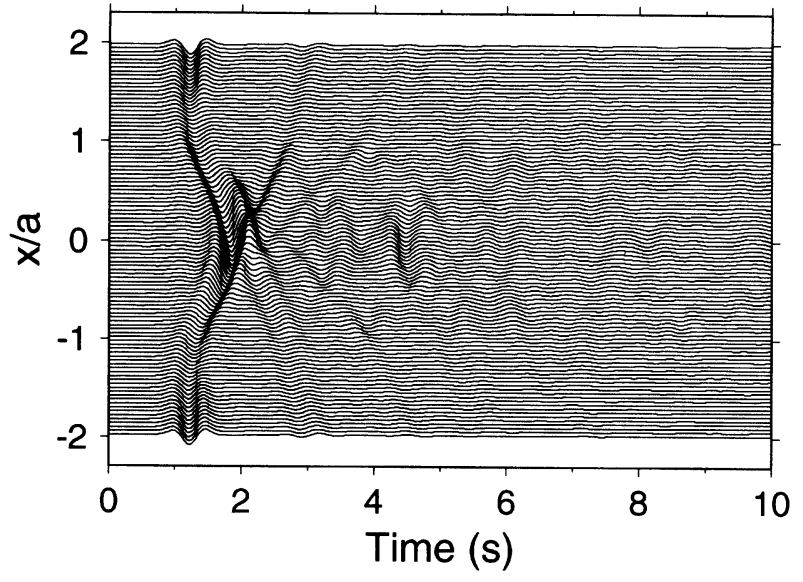

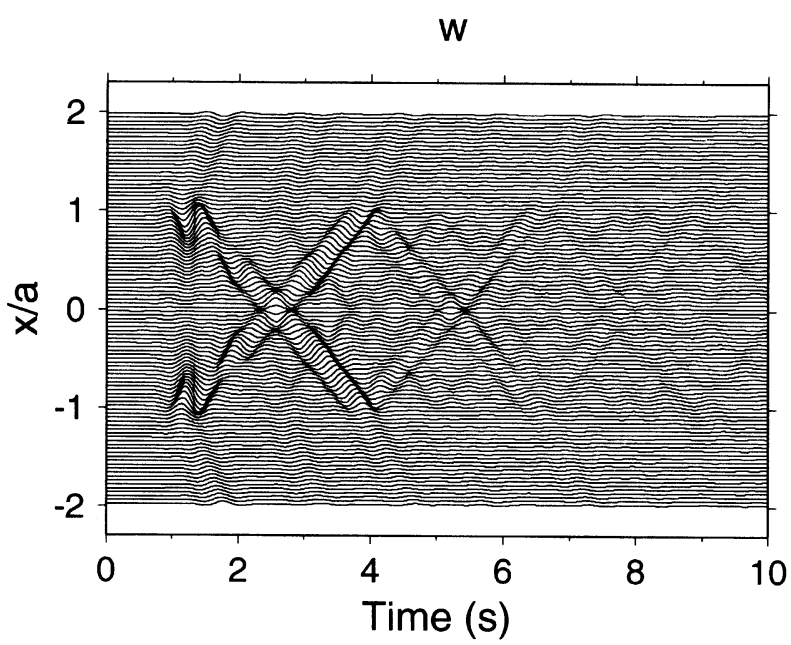

\section{(b) half-domain modelling}

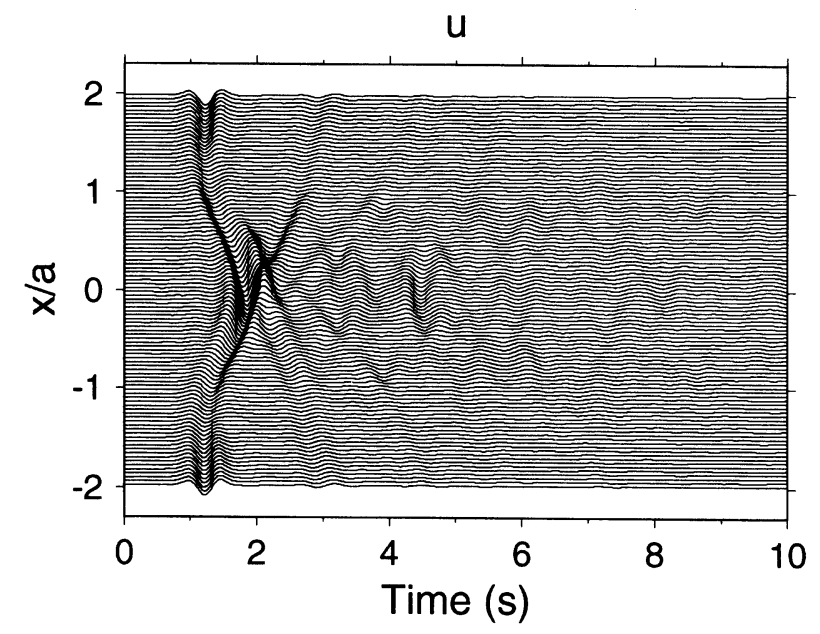

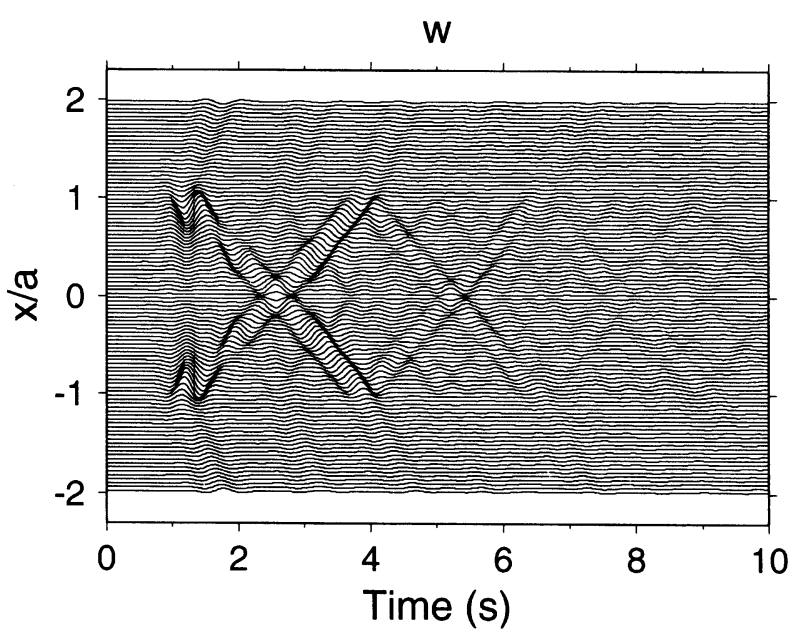

Fig. 3. Synthetic seismograms at the top of the discretised domains of the model, which is a half grid size below the free surface. (a) has been directly calculated by full-domain modelling. In (b), for $x / a>0$, the seismograms have been calculated by half-domain modelling, and the others have then been got by the symmetric and anti-symmetric extensions.

the model bounded by the plane (half-domain modelling), instead of the entire domain of the model (full-domain modelling). Furthermore, for any model symmetric with respect to two orthogonal vertical planes, the wavefield only need to be calculated in a quarter domain of the model bounded by the two planes (quarter-domain modelling).

We consider a model symmetric with respect to the plane $x=0(y-z$ plane $)$ or the plane $y=0(z-x$ plane $)$, or the two planes $x=0$ and $y=0$. For 3-D modelling, 18 spatial derivatives in Eqs. (1) and (3) are calculated at each time step, among which six $z$-derivatives, i.e. $\partial u / \partial z, \partial v / \partial z, \partial w / \partial z$, $\partial \sigma_{z x} / \partial z, \partial \sigma_{y z} / \partial z, \partial \sigma_{z z} / \partial z$, are computed by the normal Fourier differentiation scheme, while 12 derivatives with respect to the horizontal coordinates (horizontal derivatives) in the equations can be calculated by the Fourier differentiation scheme with symmetric or anti-symmetric extension or the normal Fourier differentiation scheme, chosen by the type of symmetry of the configuration. Table 1 shows the scheme to be chosen for calculating each horizontal derivative of each of the following eight cases of symmetric configurations specified by the types of medium symmetry and incident wave or force system of source, where we have assumed the source is distributed symmetrically with respect to the planes of symmetry:

Case 1-(a), where the medium parameters are symmetric with respect to the plane $x=0$, i.e.

$$
\begin{aligned}
& \lambda(-x, y, z)=\lambda(x, y, z), \\
& \mu(-x, y, z)=\mu(x, y, z), \\
& \rho(-x, y, z)=\rho(x, y, z),
\end{aligned}
$$

and the wavefield is excited by vertical incidence of a plane $P$-wave, or a vertically incident plane $S$-wave oscillating in the $y$-direction, or source with force system symmetric in the 
(a)
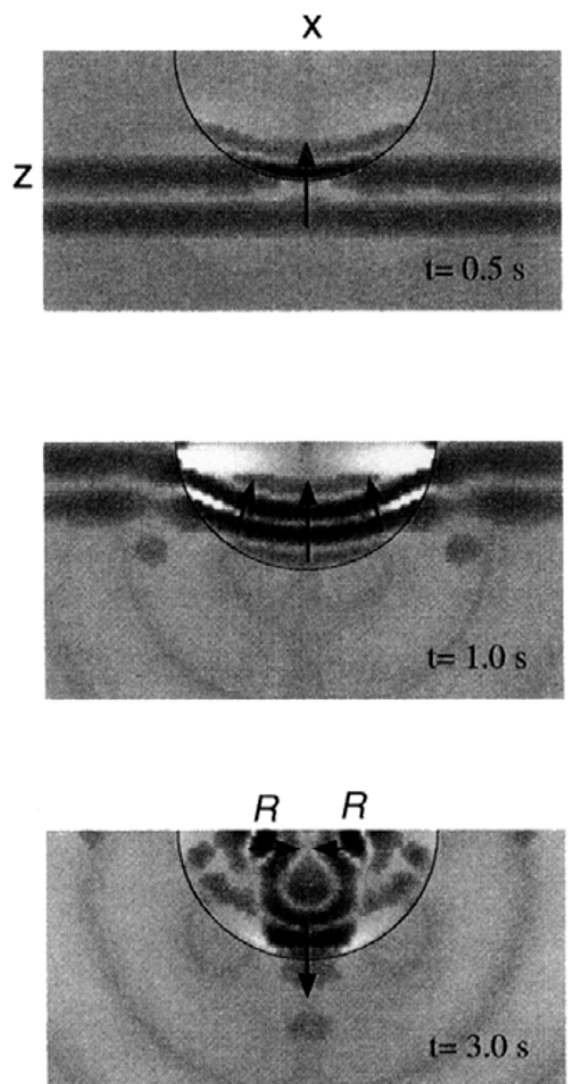

(b)
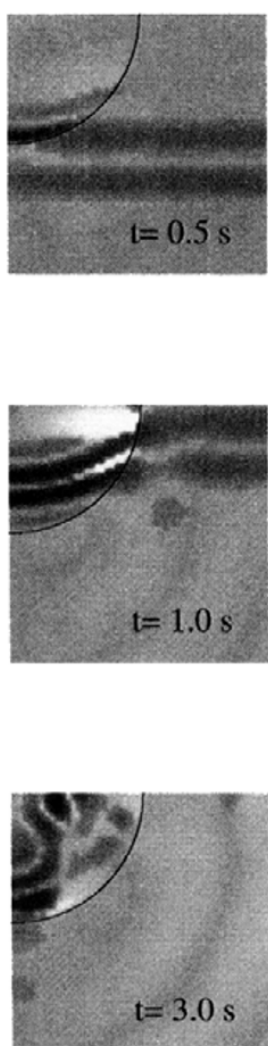

(c)
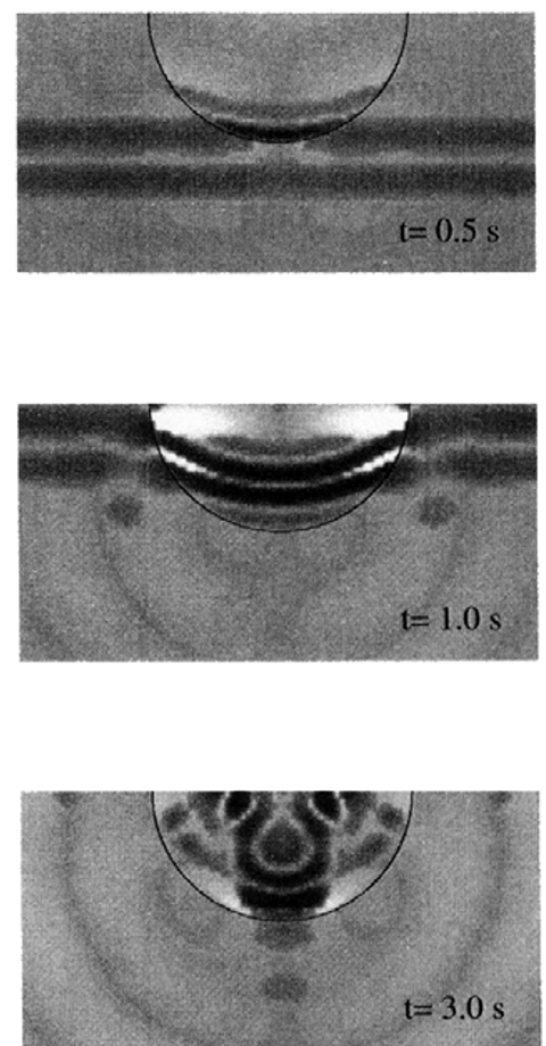

Fig. 4. Snapshots of the $S V$ and $P$ wavefield at three time steps. Black and white areas represent $S V$ and $P$ waves, respectively. (a) Entire wavefield calculated directly by full-domain modelling. (b) Wavefield calculated directly by half-domain modelling. (c) Entire wavefield derived by extension of (b). Arrows indicate propagation direction of major phases, and "R" denotes the Rayleigh wave.

$x$ coordinate, such as

$$
\left[\begin{array}{c}
0 \\
f_{y} \\
f_{z}
\end{array}\right] \text { and/or }\left[\begin{array}{ccc}
M_{x x} & 0 & 0 \\
0 & M_{y y} & M_{y z} \\
0 & M_{z y} & M_{z z}
\end{array}\right]
$$

Case 1-(b), where the medium parameters are symmetric with respect to the plane $x=0$, and the wavefield is excited by vertical incidence of a plane $S$-wave oscillating in the $x$ direction, or source with force system anti-symmetric in $x$, such as

$$
\left[\begin{array}{c}
f_{x} \\
0 \\
0
\end{array}\right] \text { and/or }\left[\begin{array}{ccc}
0 & M_{x y} & M_{x z} \\
M_{y x} & 0 & 0 \\
M_{z x} & 0 & 0
\end{array}\right]
$$

Case 2-(a), where the medium parameters are symmetric with respect to the plane $y=0$, i.e.

$$
\begin{aligned}
& \lambda(x,-y, z)=\lambda(x, y, z), \\
& \mu(x,-y, z)=\mu(x, y, z), \\
& \rho(x,-y, z)=\rho(x, y, z),
\end{aligned}
$$

and the wavefield is excited by vertical incidence of a plane $P$-wave, or a vertically incident plane $S$-wave oscillating in the $x$-direction, or source with force system symmetric in the $y$ coordinate, such as

$$
\left[\begin{array}{c}
f_{x} \\
0 \\
f_{z}
\end{array}\right] \text { and/or }\left[\begin{array}{ccc}
M_{x x} & 0 & M_{x z} \\
0 & M_{y y} & 0 \\
M_{z x} & 0 & M_{z z}
\end{array}\right] \text {; }
$$

Case 2-(b), where the medium parameters are symmetric with respect to the plane $y=0$, and the wavefield is excited by vertical incidence of a plane $S$-wave oscillating in the $y$ direction, or source with force system anti-symmetric in $y$, such as

$$
\left[\begin{array}{c}
0 \\
f_{y} \\
0
\end{array}\right] \text { and/or }\left[\begin{array}{ccc}
0 & M_{x y} & 0 \\
M_{y x} & 0 & M_{y z} \\
0 & M_{z y} & 0
\end{array}\right] \text {; }
$$

Case 3-(a), where the medium parameters are symmetric with respect to the two orthogonal planes $x=0$ and $y=0$, i.e. both Eqs. (25) and (28), and the wavefield is excited by vertical incidence of a plane $P$-wave, or source with force system symmetric both in the $x$ and $y$ coordinates, such as

$$
\left[\begin{array}{l}
0 \\
0 \\
f_{z}
\end{array}\right] \text { and/or }\left[\begin{array}{ccc}
M_{x x} & 0 & 0 \\
0 & M_{y y} & 0 \\
0 & 0 & M_{z z}
\end{array}\right] \text {; }
$$

Case 3-(b), where the medium parameters are symmetric with respect to the two planes $x=0$ and $y=0$, and the 
Table 1. Schemes for horizontal differentiation of displacement and stress.

\begin{tabular}{ccccccccccccc}
\hline Case & $\partial u / \partial x$ & $\partial u / \partial y$ & $\partial v / \partial x$ & $\partial v / \partial y$ & $\partial w / \partial x$ & $\partial w / \partial y$ & $\partial \sigma_{x x} / \partial x$ & $\partial \sigma_{y y} / \partial y$ & $\partial \sigma_{x y} / \partial x$ & $\partial \sigma_{x y} / \partial y$ & $\partial \sigma_{y z} / \partial y$ & $\partial \sigma_{z x} / \partial x$ \\
\hline 1-(a) & $\mathrm{A}$ & $\mathrm{N}$ & $\mathrm{S}$ & $\mathrm{N}$ & $\mathrm{S}$ & $\mathrm{N}$ & $\mathrm{S}$ & $\mathrm{N}$ & $\mathrm{A}$ & $\mathrm{N}$ & $\mathrm{N}$ & $\mathrm{A}$ \\
1-(b) & $\mathrm{S}$ & $\mathrm{N}$ & $\mathrm{A}$ & $\mathrm{N}$ & $\mathrm{A}$ & $\mathrm{N}$ & $\mathrm{A}$ & $\mathrm{N}$ & $\mathrm{S}$ & $\mathrm{N}$ & $\mathrm{N}$ & $\mathrm{S}$ \\
2-(a) & $\mathrm{N}$ & $\mathrm{S}$ & $\mathrm{N}$ & $\mathrm{A}$ & $\mathrm{N}$ & $\mathrm{S}$ & $\mathrm{N}$ & $\mathrm{S}$ & $\mathrm{N}$ & $\mathrm{A}$ & $\mathrm{A}$ & $\mathrm{N}$ \\
2-(b) & $\mathrm{N}$ & $\mathrm{A}$ & $\mathrm{N}$ & $\mathrm{S}$ & $\mathrm{N}$ & $\mathrm{A}$ & $\mathrm{N}$ & $\mathrm{A}$ & $\mathrm{N}$ & $\mathrm{S}$ & $\mathrm{S}$ & $\mathrm{N}$ \\
3-(a) & $\mathrm{A}$ & $\mathrm{S}$ & $\mathrm{S}$ & $\mathrm{A}$ & $\mathrm{S}$ & $\mathrm{S}$ & $\mathrm{S}$ & $\mathrm{S}$ & $\mathrm{A}$ & $\mathrm{A}$ & $\mathrm{A}$ & $\mathrm{A}$ \\
3-(b) & $\mathrm{A}$ & $\mathrm{A}$ & $\mathrm{S}$ & $\mathrm{S}$ & $\mathrm{S}$ & $\mathrm{A}$ & $\mathrm{S}$ & $\mathrm{A}$ & $\mathrm{A}$ & $\mathrm{S}$ & $\mathrm{S}$ & $\mathrm{A}$ \\
3-(c) & $\mathrm{S}$ & $\mathrm{S}$ & $\mathrm{A}$ & $\mathrm{A}$ & $\mathrm{A}$ & $\mathrm{S}$ & $\mathrm{A}$ & $\mathrm{S}$ & $\mathrm{S}$ & $\mathrm{A}$ & $\mathrm{A}$ & $\mathrm{S}$ \\
3-(d) & $\mathrm{S}$ & $\mathrm{A}$ & $\mathrm{A}$ & $\mathrm{S}$ & $\mathrm{A}$ & $\mathrm{A}$ & $\mathrm{A}$ & $\mathrm{A}$ & $\mathrm{S}$ & $\mathrm{S}$ & $\mathrm{S}$ & $\mathrm{S}$ \\
\hline
\end{tabular}

S: Fourier differentiation with symmetric extension; A: Fourier differentiation with anti-symmetric extension; N: normal Fourier differentiation.

wavefield is excited by vertical incidence of a plane $S$-wave oscillating in the $y$-direction, or source with force system symmetric in $x$ and anti-symmetric in $y$, such as

$$
\left[\begin{array}{c}
0 \\
f_{y} \\
0
\end{array}\right] \text { and/or }\left[\begin{array}{ccc}
0 & 0 & 0 \\
0 & 0 & M_{y z} \\
0 & M_{z y} & 0
\end{array}\right] \text {; }
$$

Case 3-(c), where the medium parameters are symmetric with respect to the two planes $x=0$ and $y=0$, and the wavefield is excited by vertical incidence of a plane $S$-wave oscillating in the $x$-direction, or source with force system anti-symmetric in $x$ and symmetric in $y$, such as

$$
\left[\begin{array}{c}
f_{x} \\
0 \\
0
\end{array}\right] \text { and/or }\left[\begin{array}{ccc}
0 & 0 & M_{x z} \\
0 & 0 & 0 \\
M_{z x} & 0 & 0
\end{array}\right] ;
$$

Case 3-(d), where the medium parameters are symmetric with respect to the two planes $x=0$ and $y=0$, and the wavefield is excited by source with force system anti-symmetric both in the $x$ and $y$ coordinates, such as

$$
\left[\begin{array}{ccc}
0 & M_{x y} & 0 \\
M_{y x} & 0 & 0 \\
0 & 0 & 0
\end{array}\right] .
$$

When the schemes along Table 1 can be used for a 3-D modelling, for Cases 1-(a)(b) and Cases 2-(a)(b) we can do a half-domain modelling (using the half domain $x>0$ for Cases 1-(a)(b), $y>0$ for Cases 2-(a)(b)), which can reduce the required computer memory and computation time ideally by half as compared with the full-domain modelling. Further, for Cases 3-(a)(b)(c)(d) we can do a quarter-domain modelling (using the quarter domain $x>0, y>0$ ), which can reduce the memory and computation time ideally by quarter as compared with the full-domain modelling. In case of a general force system of single force and moment tensor source, we might have to perform two independent computations corresponding to Cases 1-(a) and (b) or Cases 2-(a) and (b), or two to four independent calculations corresponding to Cases 3-(a) and/or (b) and/or (c) and/or (d), to calculate their linear combination. Then the computer memory can still be reduced by half or quarter as compared with the full-domain modelling, while the computation time might not be saved.

\section{Conclusion}

We have presented an efficient approach of the pseudospectral method for calculation of wavefields in models symmetric with respect to a vertical plane or two orthogonal vertical planes. Using this approach, the wavefields only need to be computed in a half or quarter domain of the models, so that the computer memory and computation time can be reduced ideally by half or quarter, respectively, as compared with the calculation of the entire models.

Acknowledgments. We would like to thank Takao Kagawa and the anonymous reviewer for critically reading the original manuscript and for providing helpful suggestions. This study was partially supported by the Superplume Project funded by the Science and Technology Agency.

\section{References}

Boore, D. M., Finite-difference methods for seismic wave propagation in heterogeneous materials, in Methods in Computational Physics, vol. 11, edited by B. A. Bolt, 310pp., Academic Press, New York, 1972

Cerjan, C., D. Kosloff, R. Kosloff, and M. Reshef, A non-reflecting boundary condition for discrete acoustic and elastic wave equations, Geophysics, 50, 705-708, 1985.

Daudt, C. R., L. W. Brail, R. L. Nowack, and C. S. Chiang, A comparison of finite-difference and Fourier method calculations of synthetic seismograms, Bull. Seism. Soc. Am., 79, 1210-1230, 1989.

Fornberg, B., The pseudospectral method: Comparisons with finite differences for the elastic wave equation, Geophysics, 52, 483-501, 1987.

Furumura, T. and K. Koketsu, Specific distribution of ground motion during the 1995 Kobe earthquake and its generation mechanism, Geophys. Res. Lett., 25, 785-788, 1998.

Furumura, T. and H. Takenaka, A stable method for numerical differentiation of data with discontinuities at end-points by means of Fourier transformSymmetric differentiation, Butsuri-Tansa (J. SEGJ), 45, 303-309, 1992 (in Japanese with English abstract).

Furumura, T., B. L. N. Kennett, and H. Takenaka, Parallel 3-D pseudospectral simulation of seismic wave propagation, Geophysics, 63, 279-288, 1998.

Kosloff, D., M. Reshef, and D. Loewenthal, Elastic wave calculations by the Fourier method, Bull. Seism. Soc. Am., 74, 875-891, 1984.

Reshef, M., D. Kosloff, M. Edwards, and C. Hsiung, Three-dimensional elastic modeling by the Fourier method, Geophysics, 53, 1184-1193, 1988.

Saatcilar, R. and S. Ergintav, Solving elastic wave equation with the Hartley method, Geophysics, 56, 274-278, 1991.

H. Takenaka (e-mail: takenaka@geo.kyushu-u.ac.jp), Y. Wang, and T. Furumura 